\title{
Research Paper \\ Observing the Anti-oxidant and Anti-inflammatory Effect of Nigella Sativa Com- bined With Silybum Marianum Extracts on the Acute Peritonitis Mouse Model
}

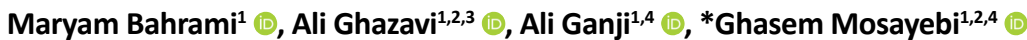

1. Department of Immunology \& Microbiology, School of Medicine, Arak University of Medical Sciences, Arak, Iran.

2. Department of Microbiology and Immunology, Traditional and Complementary Medicine Research Center (TCMRC), School of Medicine, Arak University of Medical Sciences, Arak, Iran.

3. Department of Microbiology and Immunology, Molecular and Medicine Research Center, School of Medicine, Arak University of Medical Sciences, Arak, Iran. 4. Department of Microbiology and Immunology, Infectious Diseases Research Center (IDRC), School of Medicine Arak University of Medical Sciences, Arak, Iran.

\begin{tabular}{|c|c|}
\hline $\begin{array}{l}\text { Use vour devicict to san } \\
\text { and read the article online }\end{array}$ & Citetion: Bahrami M, Ghazavi A, Ganji A, Mosayebi Gh. [Observing the Anti-oxidant and Anti-inflammatory Effect of Nige- \\
\hline arista & $\begin{array}{l}\text { 1la Sativa Combined With Silybum Marianum Extracts on the Acute Peritonitis Mouse Model (Persian)]. Journal of Arak Univer- } \\
\text { sity of Medical Sciences(JAMS). 2021; 24(3):372-385. https://doi.org/10.32598/JAMS.24.3.6154.1 }\end{array}$ \\
\hline I.Fitetis & dei https://doi.org/10.32598/JAMS.24.3.6154.1 \\
\hline
\end{tabular}

\section{(i) (8)}

Article Info:

Received: 28 Apr 2020

Accepted: 17 Apr 2021

Available Online: 01 Aug 2021

Key words:

Silybum marianum, Nigella sativa, Inflammation, Peritonitis, Antioxidant

\section{A B S TRACT}

Background and Aim In addition to free radicals such as Nitric Oxide (NO), inflammation is one of the most important pathophysiological causes of peritonitis. Over thousands of years, Nigella Sativa (NS) and Silybum Marianum (SM) are two plants known for their anti-oxidant and anti-inflammatory properties. However, the effect of its compound is unclear. Thus, in this study, we evaluated the anti-inflammatory effect of NS and SM extracts and their combination on inflammatory diseases like thioglycollate peritoneal. Methods \& Materials Alcoholic extracts of SM and NS were obtained by the soxhlet method. Male Balb/C mice were divided into 5 groups and gavage orally for 14 days with SM, NS, the mixture of extracts of these two, DMSO 30\% as the control group, and dexamethasone as the positive control group. The safety profile and acute toxicity in mice were assessed. On day 10, acute peritonitis was induced by thioglycollate $3 \%$. Finally, the total anti-oxidant power and NO concentration were measured by FRAP and Griess method, respectively, in the serum of treated mice.

Ethical Considerations All experimental process was performed following the guidelines according to the Animal Ethics Committee of Arak University of Medical Sciences (IR.ARAKMU.REC.1397.359).

Results Acute toxicity test showed no significant changes in weight and physical appearance of the mice. However, the extract and their mixture decreased NO level significantly $(P=0.000)$ in serum. Also, the mixture significantly increased total anti-oxidant power $(\mathrm{P}=0.015)$.

Conclusion Results showed that the SM and NS extract mixture demonstrated anti-inflammatory activity, inhibiting inflammatory mediators such as $\mathrm{NO}$ and increasing anti-oxidant power, thus supporting its therapeutic potential in slowing down inflammatory processes in inflammation disorders.

\section{Extended Abstract}

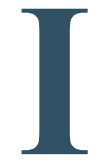

\section{Introduction}

$\mathrm{n}$ addition to inflammation, free radicals like Nitric Oxide (NO) are the most im- portant pathophysiological cause of inflammatory diseases such as peritonitis [1]. Free radicals cause extensive damage to the body's macromolecules, which are neutralized by the anti-oxidant system [2]. So, researchers started an investigation for new anti-inflammatory and anti-oxidant

\section{* Corresponding Author:}

Ghasem Mosayebi, PhD.

Address: Department of Immunology \& Microbiology, School of Medicine, Arak University of Medical Sciences, Arak, Iran

Tel: +98 (86) 34173502

E-mail: ghasemmosayebi@arakmu.ac.ir 


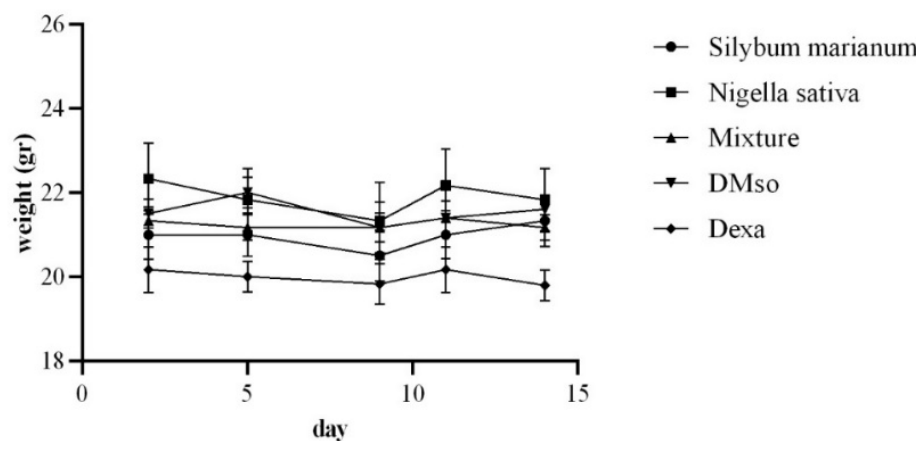

Figure 1. Acute toxicity

drugs due to the need for effective drugs with fewer side effects than chemical drugs [3].

Over thousands of years, many natural products have been used to treat various diseases, regardless of their antagonistic and synergistic effects [4]. Nigella Sativa (NS) and Silybum Marianum (SM) are promising herbal medicine in Asia used for their anti-inflammatory properties [5]. Other studies have shown that SM has anti-inflammatory and anti-oxidant properties and is not even toxic in large quantities [6-10]; however, the effect of its compound is unclear. Thus, in this study, we evaluated the anti-inflammatory effect of Nigella sativa and Silybum Marianum extracts and their combination of inflammatory diseases like thioglycollate peritoneal inflammation.

\section{Materials and Methods}

Dried SM was extracted in a Soxhlet system using n-hexane and methanol as solvent. Also, NS seeds were extracted with $95 \%$ ethanol. Then, they were filtered, and the solvent was removed in a rotary evaporator $[11,12]$. The safety profile of extracted was performed on spleen lymphocytes and acute toxicity assay according to the Organization for Economic Co-operation and Development (OECD) guidelines using the MTT assay.

Experiments were performed using male $\mathrm{Balb} / \mathrm{C}$ mice (18-22 gr). The control group received DMSO 30\% and PBS as a vehicle [13]. The positive control group gave dexamethasone $0.15 \mathrm{mg} / \mathrm{kg}$ intraperitoneal [14]. The SM and NS group were gavaged orally for 14 days. According to a previous study with $2000 \mathrm{mg} / \mathrm{kg}[15,16]$, the mixture group gavage by the SM+NS extracts was equally observed through the study [17].

To induce the peritonitis model, on day 10 of treatment, a $1 \mathrm{ml}$ sterile solution of thioglycollate medium $(3 \% \mathrm{w} / \mathrm{v}$ in PBS) was injected intraperitoneally [18]. Finally, 1214 hours after the last treatment, the mice were killed, and blood was collected from the heart to measure the total antioxidant power and $\mathrm{NO}$ concentration.

\section{NO serum}

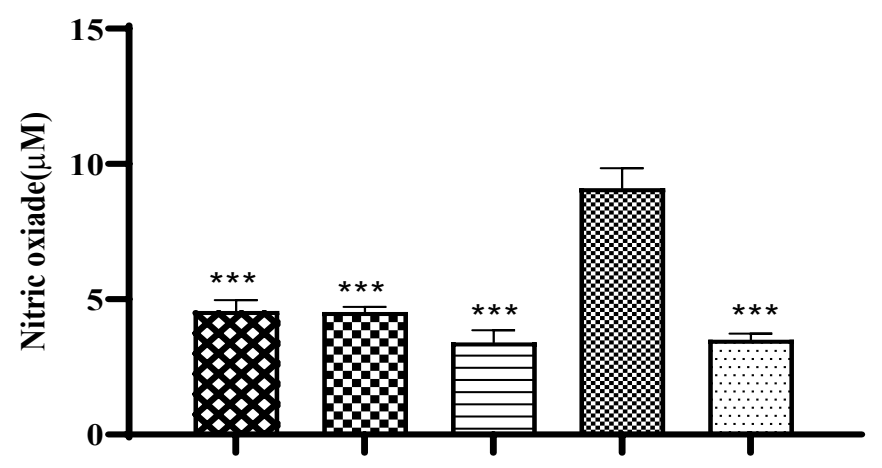

12

Silybum marianum Nigella sativa $\rightleftharpoons$ Mixture

$\Leftrightarrow$ Control Dexa

Figure 2. Nitric oxide concentration 


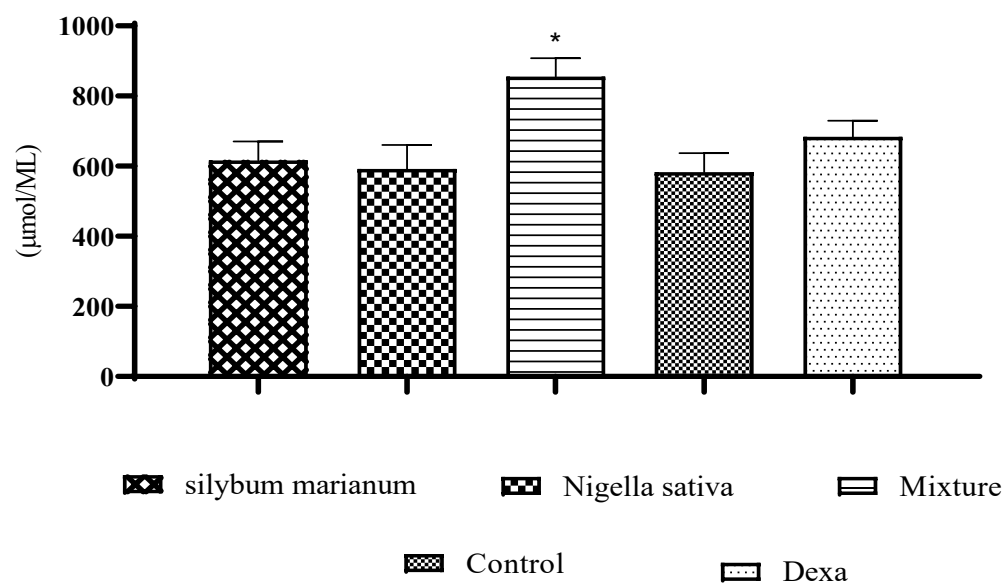

Figure 3. Anti-oxidant power

The FRAP method was based on that of Benzie and Strain [19]. Briefly, FRAP reagent (included: $300 \mathrm{mmol} / \mathrm{L}$ acetate buffer, $10 \mathrm{mmol} / \mathrm{L}$ TPTZ/HCL solution, and $20 \mathrm{mmol} / \mathrm{L}$ ferric chlorides) was added to $100 \mu \mathrm{l}$ of diluted serum. After incubation, time absorbance was measured directly at $620 \mathrm{~nm}$. The NO was measured by the Griess reaction [20]. Briefly, $100 \mu$ diluted serum of treated mice was incubated with $100 \mu \mathrm{L}$ of Griess reagent; finally, nitrite levels were measured at $620 \mathrm{~nm}$ after incubation (Figure 1, 2 \& 3).

\section{Results}

Ethanolic Extract of NS (NEE) and Methanolic extract of SM (SME) exhibited no cytotoxic effects on spleen lymphocytes. Also, the acute toxicity test showed no significant changes in weight and physical appearance of the mice. These extract and their mixture decrease NO level significantly $(\mathrm{P}=0.000)$ in the treated group compared to the control group. Also, the mixture group increased significantly total anti-oxidant power $(\mathrm{P}=0.015)$ compared to the control and each extract individually.

However, the anti-inflammatory effect of NEE and SME was shown in previous studies [21]. No changes in the weight of treated mice, with a mentioned dosage of extracts and no apparent toxicity on splenic lymphocytes, were seen. So results reveal the non-toxic nature of extract at the tested dosage consistent and, the inhibitory effects were not due to toxicity and cell killing $[22,23]$. In parallel with our study, the SME and the NEE had no acute and chronic toxicity on laboratory animals, even higher doses [24, 25].

\section{Discussion and Conclusion}

It seems that the significant increase in anti-oxidant power of the combination group against each of these extracts and the control group indicates the synergistic effect of all flavonoids in the anti-oxidant capacity of the extract [26, 27]. In confirmation, NS oil and its fractions (neutral lipids, glycolipids, and phospholipids) showed anti-oxidant activity related to their entire content [28]. In parallel, the anti-oxidant power of NS oil and SM seeds oil were reported equally [27].

Shahin et al. Showed that NS extract has more anti-oxidant effect than SM [29]. This difference can be due to the different solvents used in the extraction and confirming the role of alcoholic solvents in anti-oxidant power. According to our data, each mentioned extract and their combination has significantly decreased NO production release.

Similarly, a study showed suppression of NO production by aqueous extract of NS in stimulated peritoneal macrophages [23]. The reduction of NO production in the inflammatory model of rheumatoid arthritis in mice can confirm our results [30]. Another study showed that the extract of SM and silibinin (one of its main ingredients) reduced the NO production by blocking $\mathrm{p} 38, \mathrm{MAPK}$, or NF- $\mathrm{BB}$ signaling pathways in RAW 264.7 cells and peritoneal macrophages, which have been in line with its anti-inflammatory effects [31].

\section{Ethical Considerations}

\section{Compliance with ethical guidelines}

All experimental process was performed following the guidelines according to the Animal Ethics Committee of 
Arak University of Medical Sciences (Code: IR.ARAKMU. REC.1397.359).

Funding

This article is taken from a research project with the code 3196 and it was funded by the Vice Chancellor for Research and Technology of Arak University of Medical Sciences.

\section{Authors' contributions}

All authors met the writing standards based on the International Committee of the Journal of Medical Journalists (ICMJE).

\section{Conflicts of interest}

The authors declared no conflicts of interest.

\section{Acknowledgements}

The authors would like to thank the Deputy for Research and Technology of Arak University of Medical Sciences for their valuable support. 
This Page Intentionally Left Blank 
مقايسه اثر ثوام آثتى اكسيدانى و ضدالتهابي عصارههاي الكلى خارمريم و سياهداثه در مدل موشى بريتونيث ماده

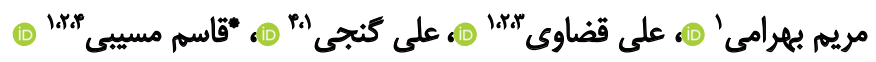

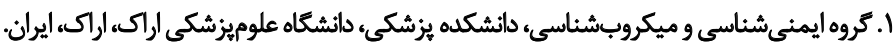

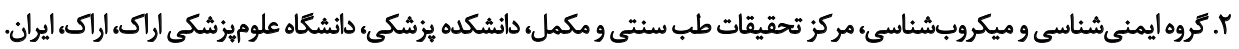

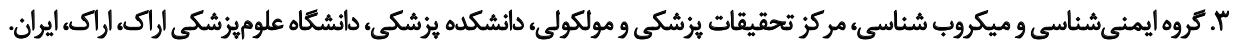

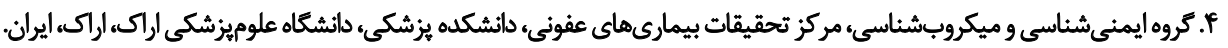

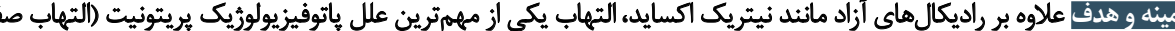

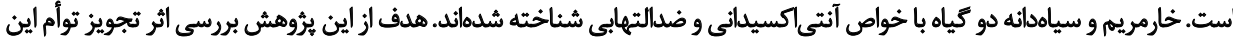

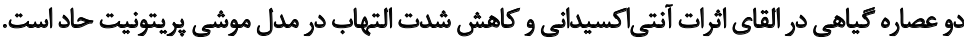

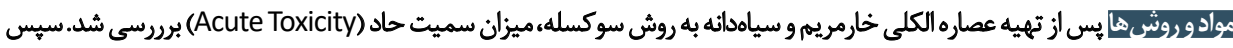

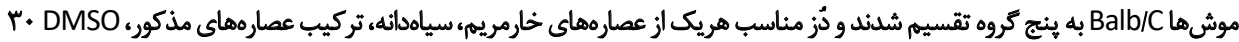

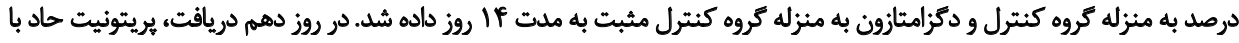

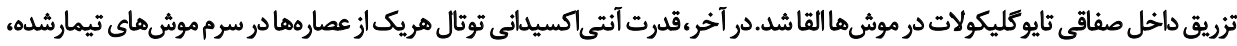

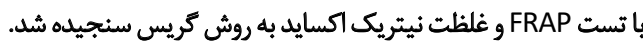

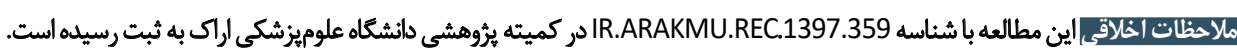

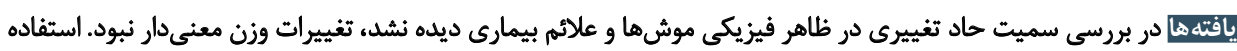

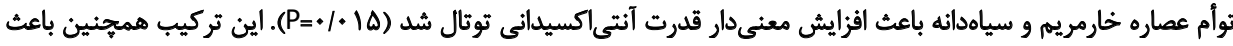

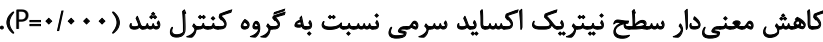

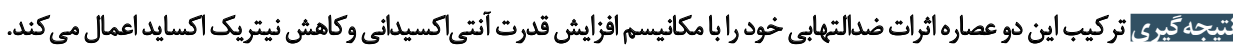

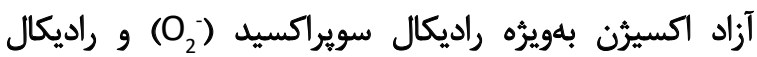

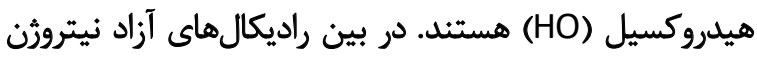

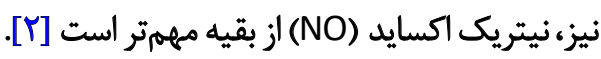

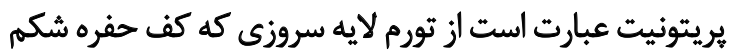

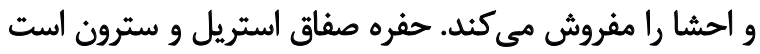

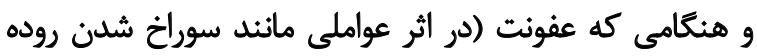

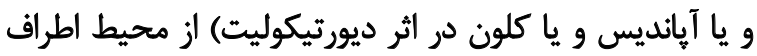

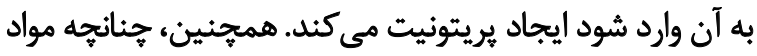

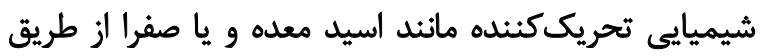

التهاب نقش كليدى در ياتوزثز بسيارى از بيمارىها از جمله

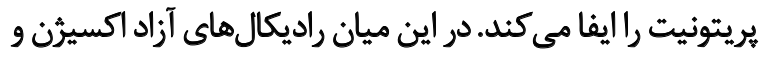

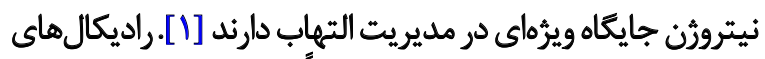

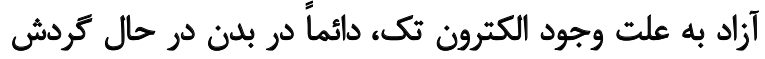

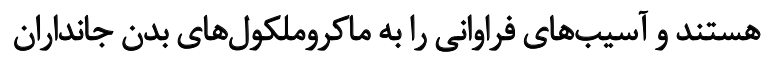

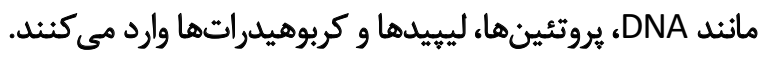

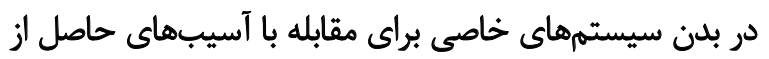

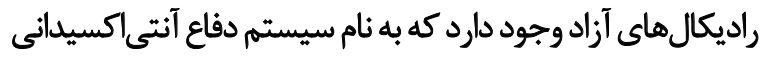

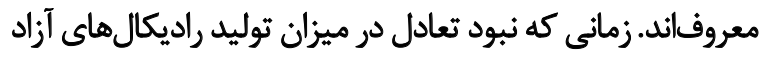




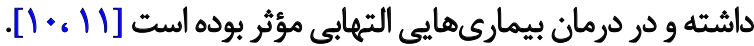

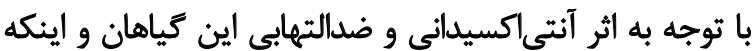

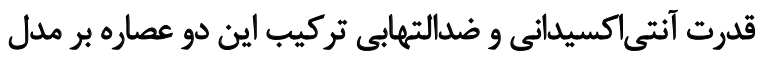

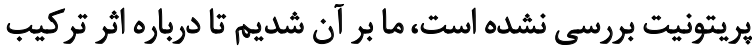
اين دو كياه را يُووهش كنيم.

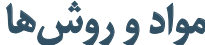

\section{illow dut}

ابتدا كياهان خارمريم و سياهدانه از مراكز معتبر تهيه شد و يس

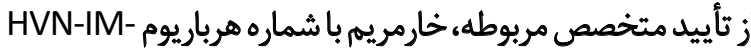

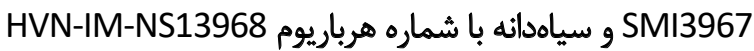

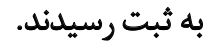

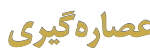

يس از تهيه كياهان خارمريم و سياهدانه از مراكز معتبر، عصاره

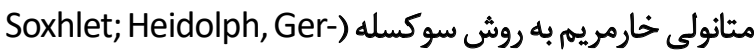
تهany

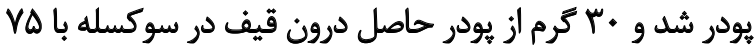

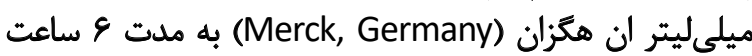

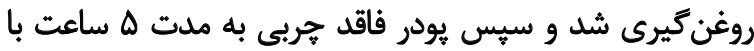

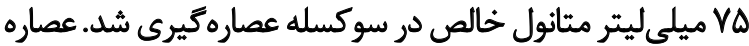

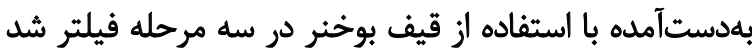

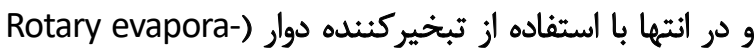
(tor; Heidolph, Germany

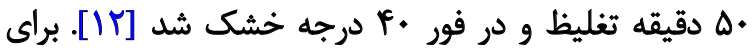

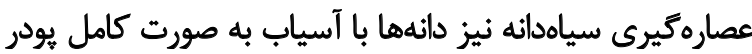

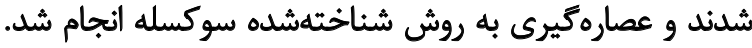

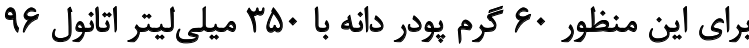

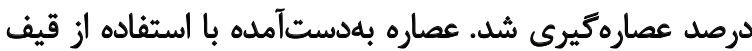

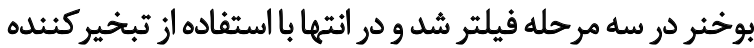

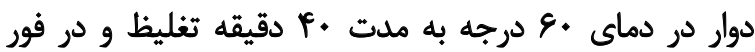

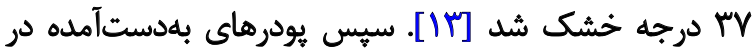

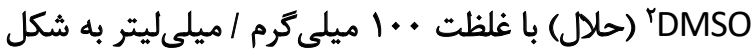

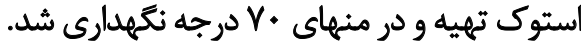

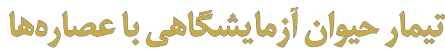

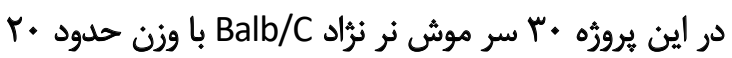

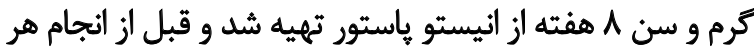

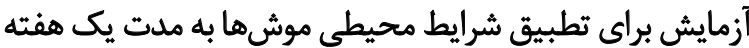

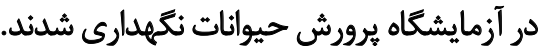

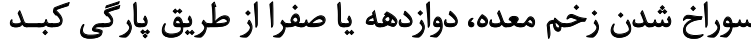

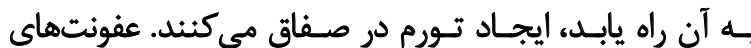

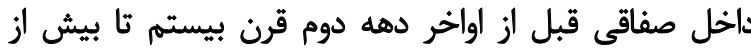

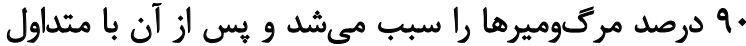

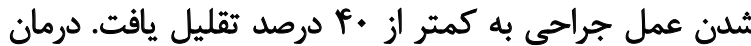

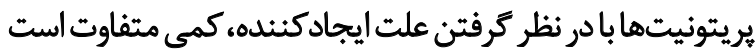

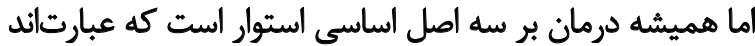

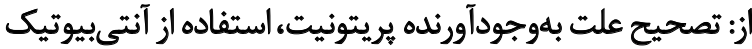

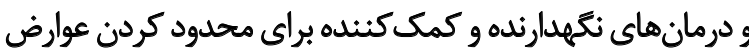

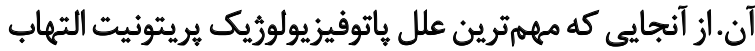

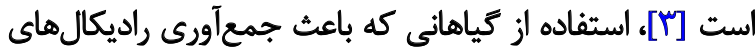

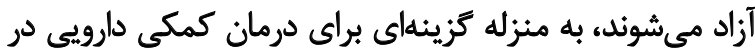

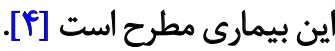
كياهان الرويى ويزّكى هايى از قبيل در دسترس بودن، سميت

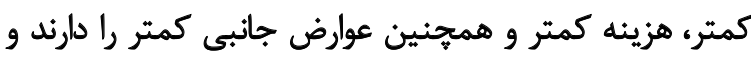

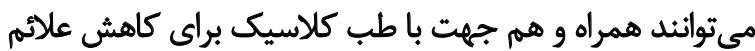

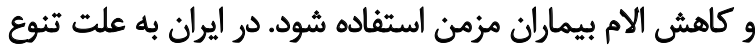

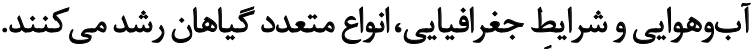

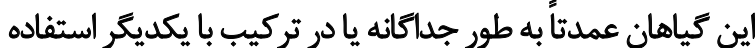

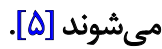

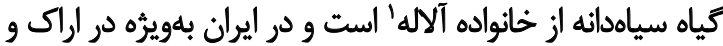

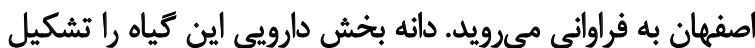

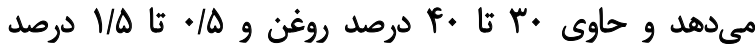
اسانس است. ماده مؤثر در سياهدانه را تيموكينون تشكيل مي تهديد

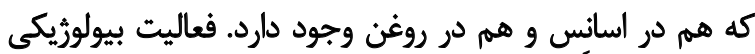

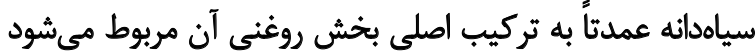

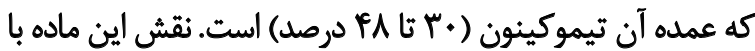

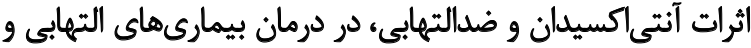

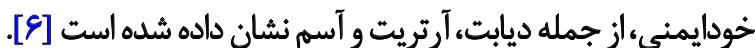

خارمريم با نام علمى Silybum marianum، كياهى يك يادو

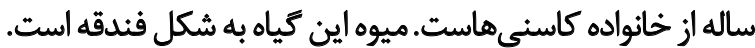

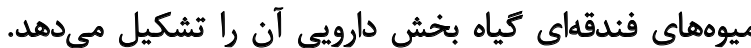

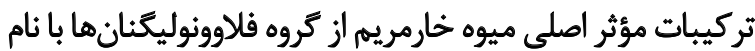

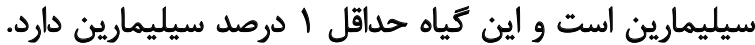

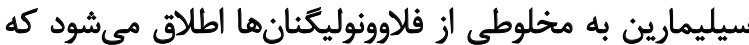

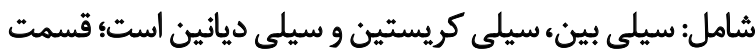

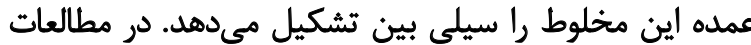

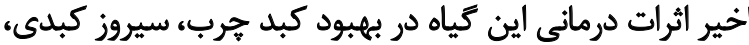

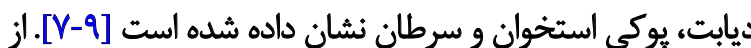

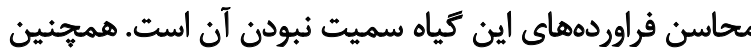
در طب ايرانى به منزله يك ضدالتهاب وآنياه ستئىاكسيدان كاربرد 


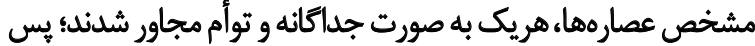

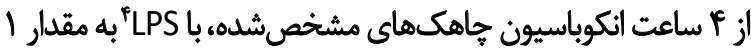

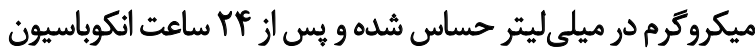

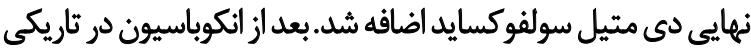
جذب نورى با استفاده از دستكاه الايزا ريدر مورد سنجش قائ قرار كرفت

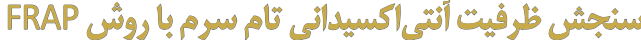

روش FRAP روشى براى اندازهكيرى ظرفيت آنتىاكسيدانى است. دراين

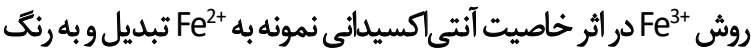

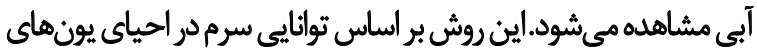
Fe $F^{3+}$ (فريك) به (فرو) در حضور مادمالى به نام

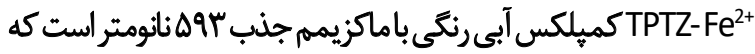

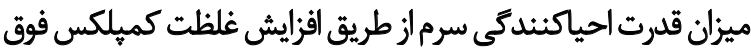

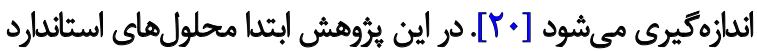

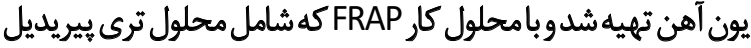

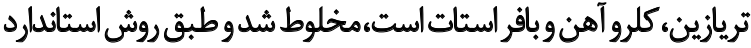
جذب آنهابا استفاده از دستكاه الايزاريدر مدل Stat Fax ساخت كشور

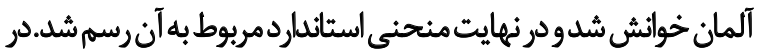

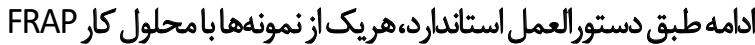

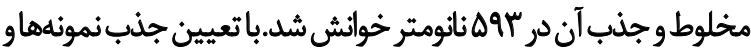

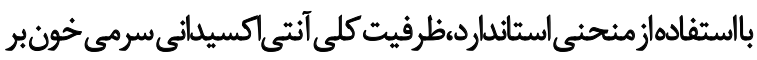
مبناي غلظت يون فرو بر حسب ميكرومول در ليتر مشخص شاندا

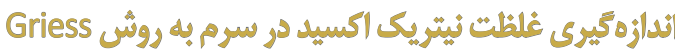

نيتريك اكسيد (NO) يكى از عوامل مهم فيزيولوئوريك و مؤثر در

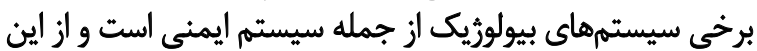

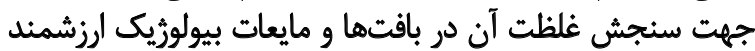

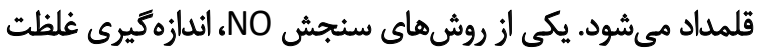

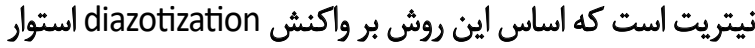

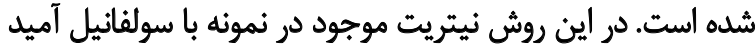

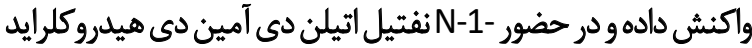
(NED)

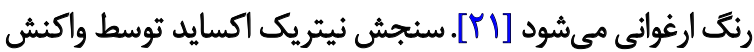

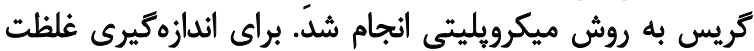

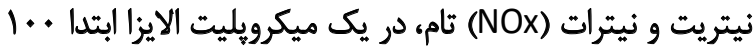

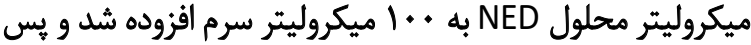

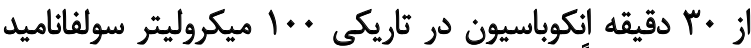

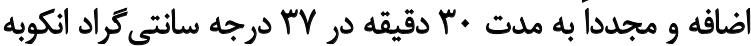

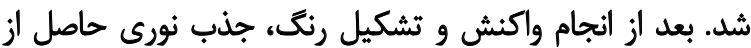

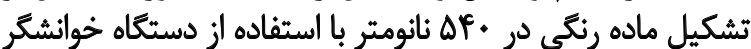

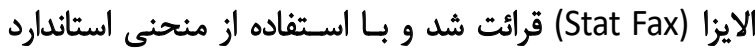
غلظت نمونهها محاسبه شد.

4. Lipopolysaccharides

5. Ferric ion reducing antioxidant power

6. Triphenyl tetrazolium
(Acute toxicity) تسيت سميثت هاد

طبق دستورالعمل OECD" براى سنجش سميت حاد، موشها

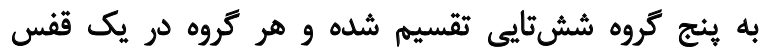

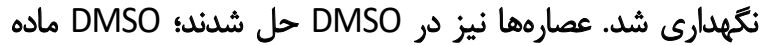

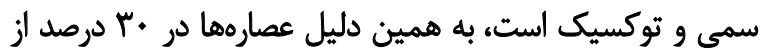

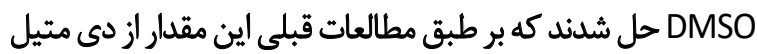

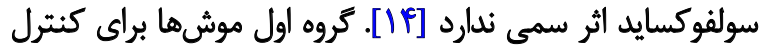

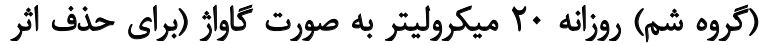

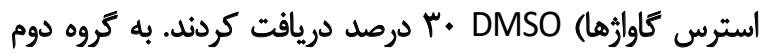

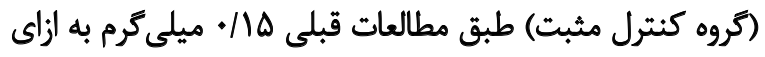

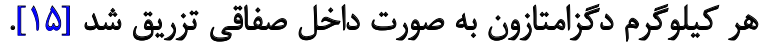

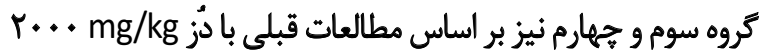

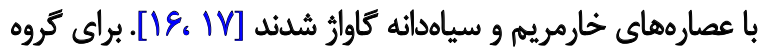

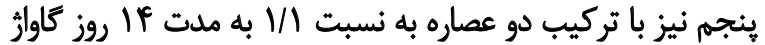

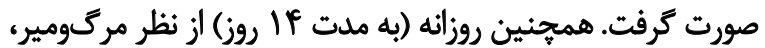

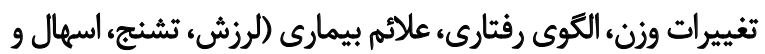

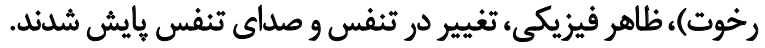

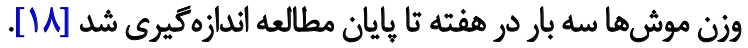

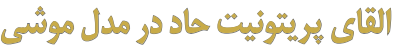

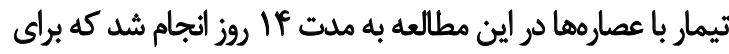

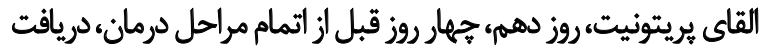

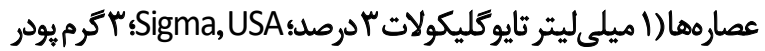

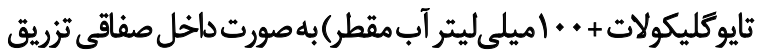

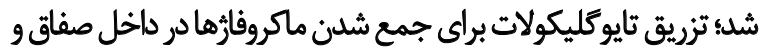

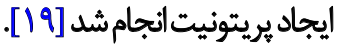

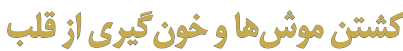

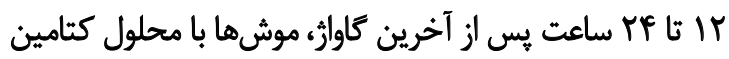

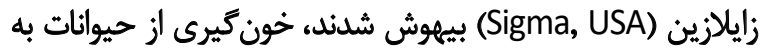

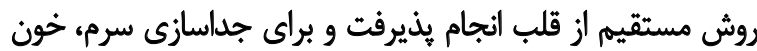

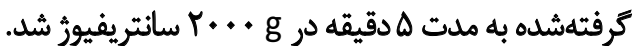

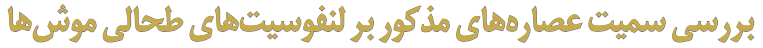

در اين راستا، پِ از خارج كردن طحال در شرايط استريل از

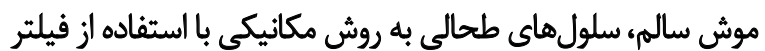
(Mesh)

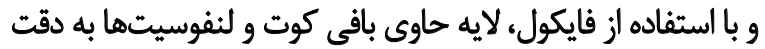

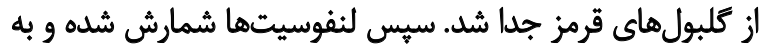

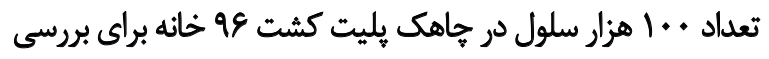

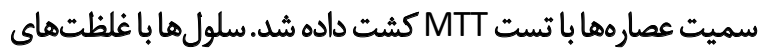

3. Organization for Economic Co-operation and Development 


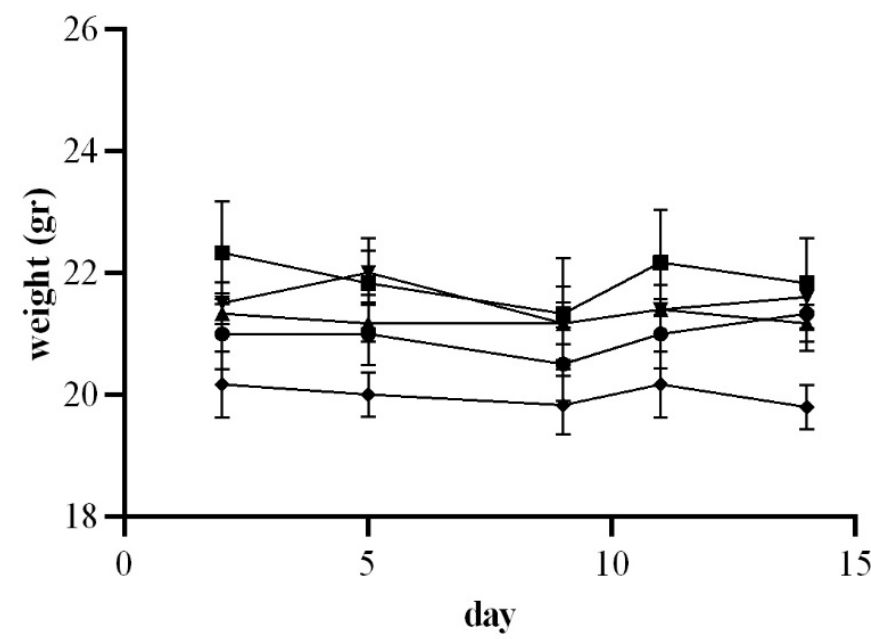

$$
\begin{aligned}
& \rightarrow \text { Silybum marianum } \\
& \rightarrow \text { Nigella sativa } \\
& \rightarrow \text { Mixture } \\
& \rightarrow \text { DMso } \\
& \rightarrow \text { Dexa }
\end{aligned}
$$

تصوير ا. تغييرات وزنى موشها در دوره مطالعه

براى بررسى سميت عصار مهاى مذكور نيز درصد حيات سلولى در

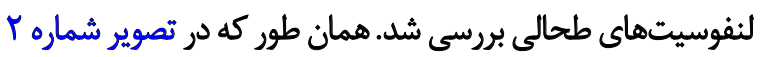

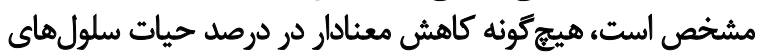

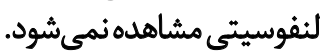

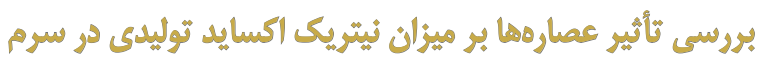

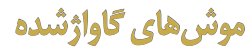
ميزان توليد نيتريت در سرم موشهاى كاوارشارده با عصارههاى

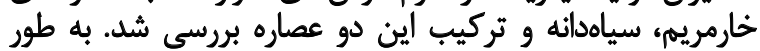

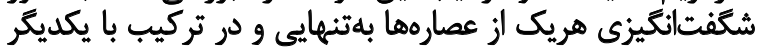

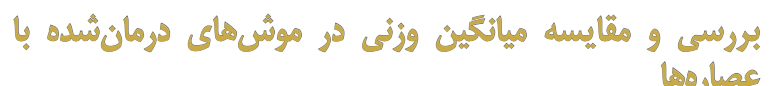
همانطور كه در تصوير شماره ا نشان داده شده است، تغيير

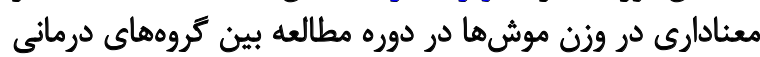

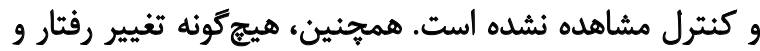

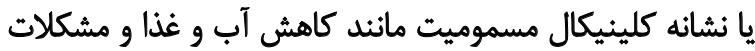

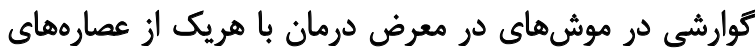

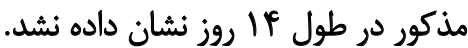

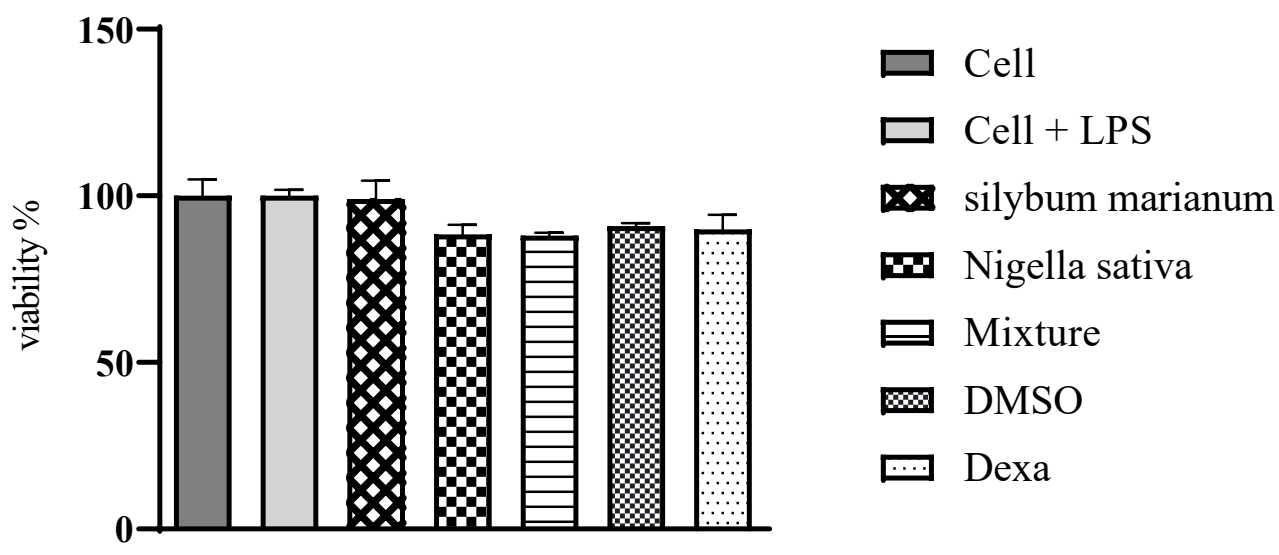

$\operatorname{LPS}(1 \mu \mathrm{g} / \mathrm{ml})+\quad+\quad+\quad+\quad++$

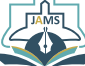

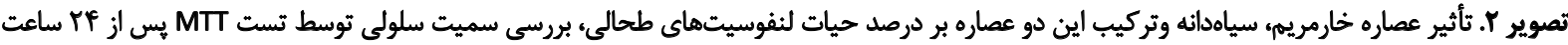

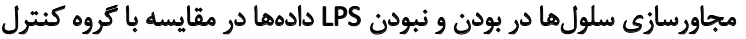




\section{NO serum}

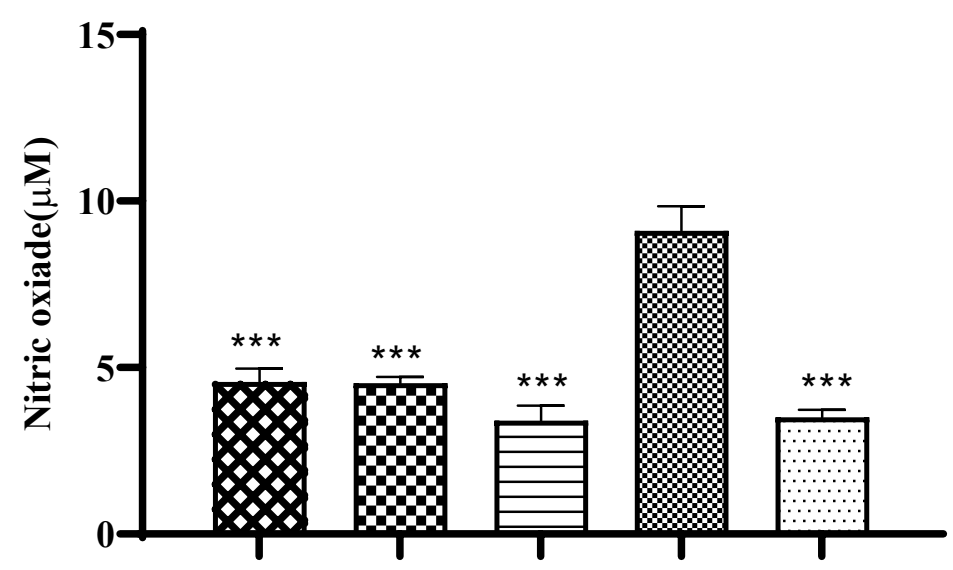

Wilybum marianum $\mathbf{W}$ Nigella sativa $\square$ Mixture

sing

\section{m Control Dexa}

تصوير ب. تأثير عصار ههاي خارمريمه، سياهدانه و تركيب اين دو عصاره به يك نسبت در توليد نيتريت در سرم موش هاى كاوارشده با هريك از عصارهها به صورت تنها و در تركيب با يكديكر در مقايسه با كروه كنترل، "* 1.

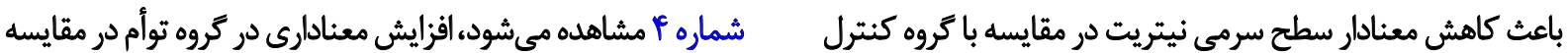

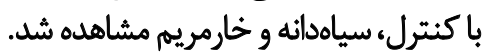

شده است (تصوير شيماره سم).

\section{بحث

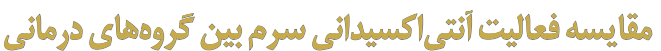

يافتن يك عامل تأثيركذار مانئ استفاده از عصارههاى كياهى

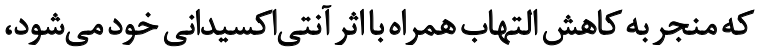

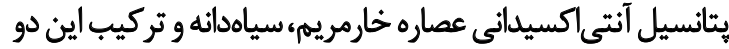
عصاره در تست FRAP بررسى و مقايسه شد. همان طور كه در تصوير

FRAP

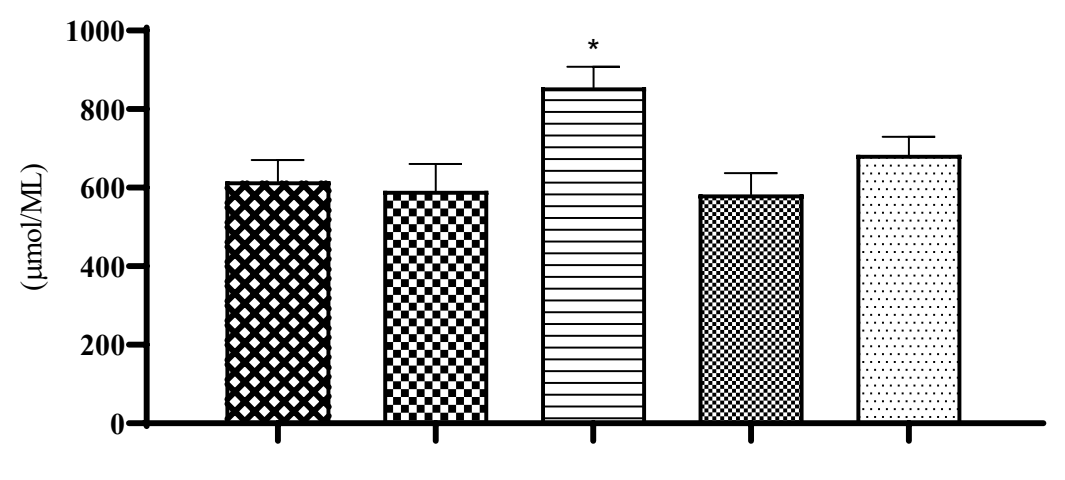

silybum marianum

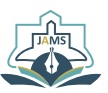

\footnotetext{
Control

Dexa

$$
\begin{aligned}
& \text { تصوير F. قدرت آنتى اكسيدانى عصاره خارمريهء سياهدانه و تركيب عصار هها به نسبت برابر در سطح سرم }
\end{aligned}
$$

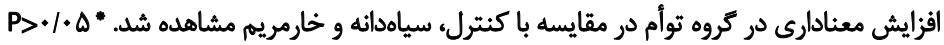


ضدالتهابى ماده مؤثر در مطالعات كذشته بوده است. مطالعات قبلى ميكي

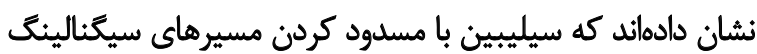

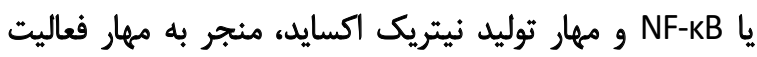

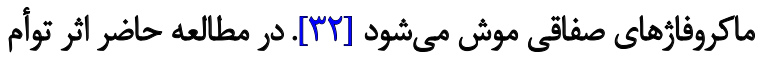

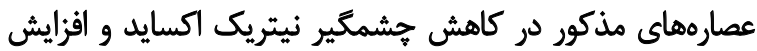

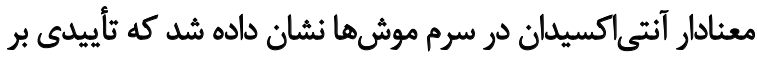

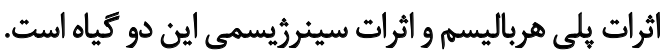

\section{تثيجنهيرى}

با توجه به دادههاى آوردمشده نتيجه كرفته مىشود كه فرضيه

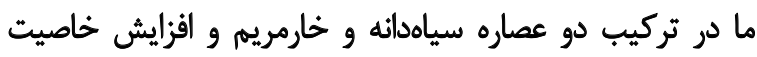

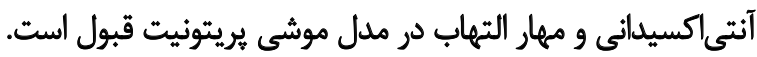
و ممكن است در جلوكيرى أز التهاب و مزمن شدن من آن آن نقش داشيته

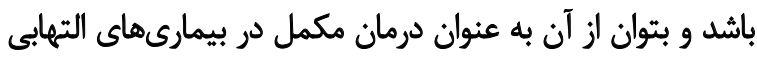

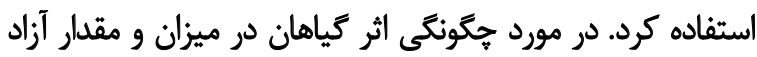
شدن واسطههاي التهابي دركير بايد تحقيقات ايمونولوريكي تكميلي

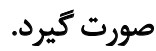

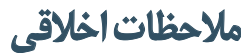

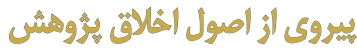

كميثه نظارث بر حقوق حيوانات آزمايشكاهى مركز تحقيقات

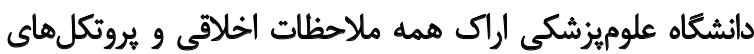

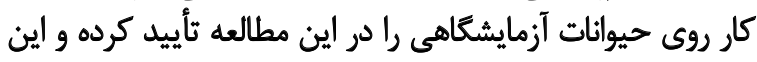
يثروهش با كد اخلاق

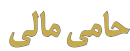

اين مقاله بركرفته از يروره تحقيقاتى با كد 19 اب است واست و هزينه انجام آن را معاونت تحقيقات و فناورى دانشكاه علوميزئشكى ارئى ياك تأمين كرده است.

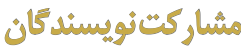

تمامى نويسندكان به يك اندازهدر نغارش مقاله مشاركت داشتهاند.

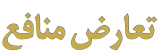

طبق نظر نويسندكان هيجكونه تضاد منافعى در برؤوه حاضر

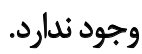

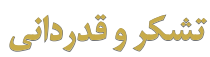

بدينوسيله از معاونت تحقيقات و شوراى ثيروهشى دانشكاه علوميزشكى اراى به دليل تأمين مالى تشكر و قدردانى مئى ئشود.
مي تواند يك درمان مكمل و حمايتي مؤثر در بيمارى هاى التهابى

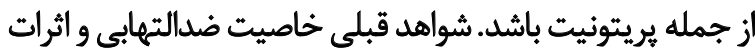

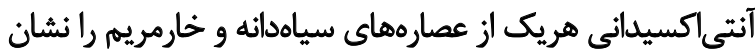

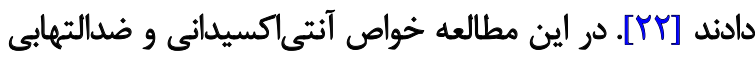

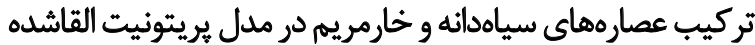
با تايوكليكولات مطالعه شد.

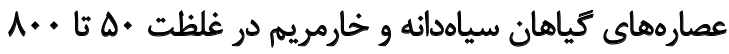

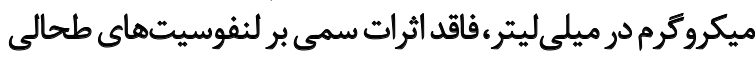

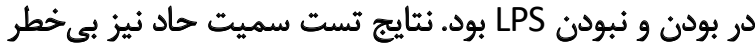

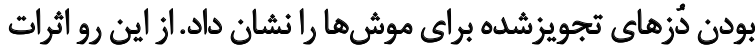

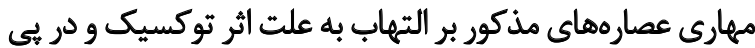

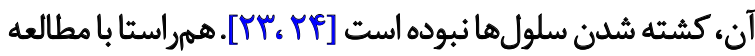

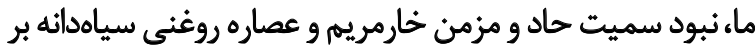

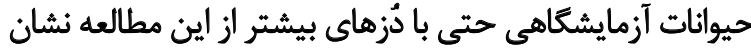

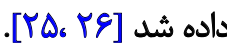

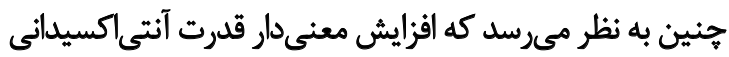

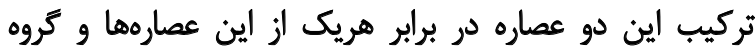

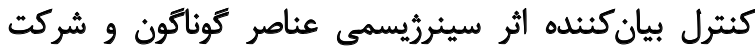

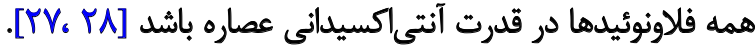

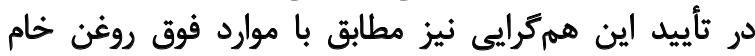

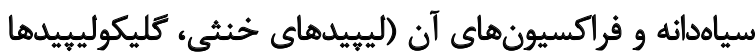

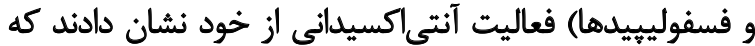

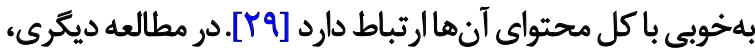

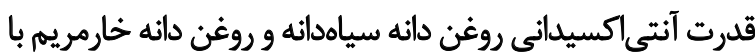

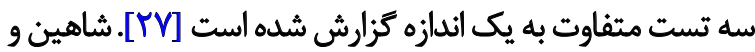
همكاران نشان دادند، سياهدانه داراي اثر آنتى اكسيداني بـاني بيشترى

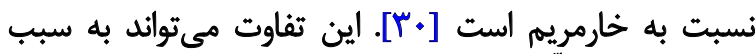

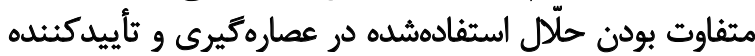
نقش حلال هاى الكلى در قدرت آنتىاكسيدانى باشدا.

با توجه به آزاد شدن واسطههاي التهابي در التهاب حاد به به

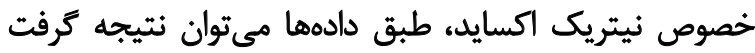

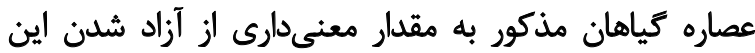

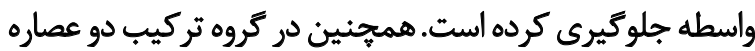

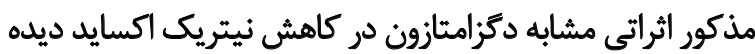

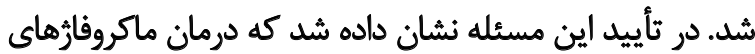

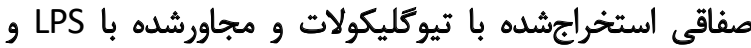

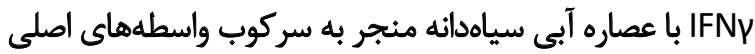

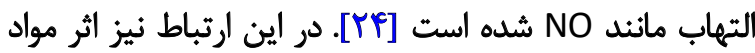

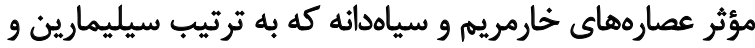

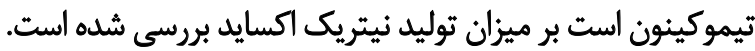

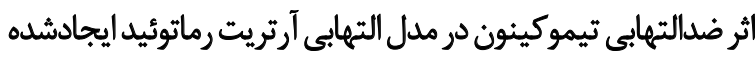

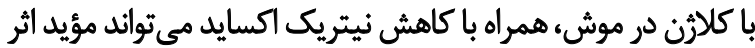

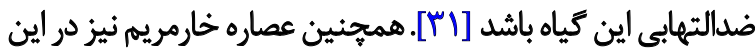
مطالعه باعث كاهش نيتريك اكسايد شده است كه همراستا با باثئ آثرات 


\section{References}

[1] Ganji A, Farahani I, Palizvan MR, Ghazavi A, Ejtehadifar M, Ebrahimimonfared $M$, et al. Therapeutic effects of walnut oil on the animal model of multiple sclerosis. Nutr Neurosci. 2019; 22(3):215-22.[DOI: 10.1080/1028415X.2017.1371389]

[2] Ghazavi A, Mosayebi G, Solhi H, Rafiei M, Moazzeni SM. Serum markers of inflammation and oxidative stress in chronic opium (Taryak) smokers. Immunol Lett. 2013; 153(1-2):22-6. [DOI:10.1016/j. imlet.2013.07.001]

[3] Jones RS, Claridge JA. Acute abdomen. In: Townsend CM, Sabiston DC, editors. Sabiston Textbook of Surgery, 17th edition. Philadelphia: Elsevior SAounders; 2004. https://books.google.com/books?id=8b_ IRwAACAAJ\&dq

[4] Mion CM, Béraud JJ. Treatment of acute renal failure by peritoneal dialysis. In Acute Renal Failure. Boston: Springer; 1984. https://link springer.com/chapter/10.1007/978-1-4613-2841-4_24

[5] Amirghofran Z. Medicinal plants as immunosuppressive agents in traditional Iranian medicine. Iran J Immunol. 2010; 7(2):65-73. [PMID]

[6] Woo CC, Kumar AP, Sethi G, Tan KHB. Thymoquinone: Potential cure for inflammatory disorders and cancer. Biochem Pharmacol. 2012 83(4):443-51. [DOI:10.1016/j.bcp.2011.09.029][PMID]

[7] Lirussi F, Beccarello A, Zanette G, De Monte A, Donadon V, Velussi $M$, et al. Silybin-beta-cyclodextrin in the treatment of patients with diabetes mellitus and alcoholic liver disease. Efficacy study of a new preparation of an anti-oxidant agent. Diabetes Nutr Metab. 2002; 15(4):222-31. [PMID]

[8] Agrawal S, Bonkovsky HL. Management of nonalcoholic steatohepatitis: An analytic review. J Clin Gastroenterol. 2002; 35(3):253-61. [DOI:10.1097/00004836-200209000-00011]

[9] Lucena MI, Andrade RJ, de la Cruz JP, Rodriguez-Mendizabal M, Blanco E, de la Cuesta FS. Effects of silymarin MZ-80 on oxidative stress in patients with alcoholic cirrhosis. Int J Clin Pharmacol Ther. 2002; 40(1):2-8. [DOI:10.5414/CPP40002]

[10] Tavakkoli A, Mahdian V, Razavi BM, Hosseinzadeh H. Review on clinical trials of black seed (Nigella sativa) and its active constituent, thymoquinone. J Pharmacopuncture. 2017; 20(3):179-93. [DOI:10.3831/KPI.2017.20.021] [PMID] [PMCID]

[11] Esmaeil N, Balouchi Anaraki S, Gharagozloo M, Moayedi B. Silymarin impacts on immune system as an immunomodulator: One key for many locks. Int Immunopharmacol. 2017; 50:194-201. [DOI:10.1016/j.intimp.2017.06.030]

[12] Wianowska D, Wiśniewski M. Simplified procedure of silymarin extraction from silybum marianum L. Gaertner. J Chromatogr Sci. 2015; 53(2):366-72.[DOI:10.1093/chromsci/bmu049]

[13] Koshak AE, Yousif NM, Fiebich BL, Koshak EA, Heinrich M. Comparative immunomodulatory activity of Nigella sativa I. preparations on proinflammatory mediators: A focus on asthma. Front Pharmacol. 2018; 9:1075. [PMID] [PMCID]

[14] Noel PRB, Barnett KC, Davies RE, Jolly DW, Leahy JS, Mawdesley$\mathrm{E}$, et al. The toxicity of dimethyl sulphoxide (DMSO) for the dog, pig, rat and rabbit. Toxicol. 1975; 3(2):143-69. [DOI:10.1016/0300$483 \times(75) 90081-5]$

[15] Xu T, Qiao J, Zhao L, He G, Li K, Wang J, et al. Effect of dexamethasone on acute respiratory distress syndrome induced by the H5N1 virus in mice. Eur Respir J. 2009; 33:852-60. [DOI:10.1183/09031936.00130507]
[16] Vahdati-Mashhadian N, Rakhshandeh H, Omidi A. An investigation on LD50 and subacute hepatic toxicity of Nigella sativa seed extracts in mice. Int J Pharm Sci. 2005; 60(7):544-7. https://www.ingentaconnect.com/content/govi/pharmaz/2005/00000060/00000007/art00013

[17] Soufy NI. Hepatoprotective and antioxidant effects of Silybum marianum plant against hepatotoxicity induced by carbon tetrachloride in rats. J Am Sci. 2012; 8(4):479-86. http://www.jofamericanscience.org/journals/am-sci/am0804/064_8482am0804_479_486.pdf

[18] Kitano M. Updating of OECD guidelines for the testing of chemicals. Wat Sci Tech. 1992; 25(11):465-72. https://www.proquest.com/ docview/1943169657

[19] Lam D, Harris D, Qin Z. Inflammatory mediator profiling reveals immune properties of chemotactic gradients and macrophage mediator production inhibition during thioglycollate elicited peritoneal inflammation. Mediators Inflamm. 2013; 1-9. [DOI:10.1155/2013/931562]

[20] Benzie IFF, Strain JJ. The Ferric Reducing Ability Of Plasma (FRAP) as a measure of "antioxidant power": The FRAP assay. Anal Biochem 1996; 239(1):70-6.[DOI:10.1006/abio.1996.0292]

[21] Green LC, Wagner DA, Glogowski J, Skipper PL, Wishnok JS, Tannenbaum SR. Analysis of nitrate, nitrite, and $\left[{ }^{15} \mathrm{~N}\right]$ nitrate in biological fluids. Anal Biochem. 1982; 126(1):131-8. [DOI:10.1016/00032697(82)90118-X]

[22] Gholamnezhad Z, Keyhanmanesh R, Boskabady MH. Anti-inflammatory, antioxidant, and immunomodulatory aspects of Nigella sativa for its preventive and bronchodilatory effects on obstructive respiratory diseases: A review of basic and clinical evidence. J Funct Foods. 2015; 17:910-27. [DOI:10.1016/j.jff.2015.06.032]

[23] Gharagozloo M, Jafari S, Esmaeil N, Javid EN, Bagherpour B, Rezaei A Immunosuppressive effect of silymarin on mitogen-activated protein kinase signalling pathway: The impact on T cell proliferation and cytokine production. Basic Clin Pharmacol Toxicol. 2013; 113(3):209-14. [DOI:10.1111/bcpt.12088]

[24] Majdalawieh AF, Hmaidan R, Carr RI. Nigella sativa modulates splenocyte proliferation, Th1/Th2 cytokine profile, macrophage function and NK anti-tumor activity. J Ethnopharmacol. 2010; 131(2):268-75 [DOI:10.1016/j.jep.2010.06.030]

[25] Hajhashemi V, Ghannadi A, Jafarabadi H. Black cumin seed essential oil, as a potent analgesic and antiinflammatory drug. Phytothe Res. 2004; 18(3):195-9. [DOI: 10.1002/ptr.1390]

[26] Bahmani M, Shirzad H, Rafieian S, Rafieian-Kopaei M. Silybum marianum: Beyond hepatoprotection. J Evid Based Complementary Altern Med. 2015; 20(4):292-301. [DOI:10.1177/2156587215571116]

[27] Meddeb W, Rezig L, Zarrouk A, Nury T, Vejux A, Prost M, et al. Cytoprotective activities of milk thistle seed oil used in traditional tunisian medicine on 7-ketocholesterol and 24s-hydroxycholesterolinduced toxicity on $158 \mathrm{~N}$ murine oligodendrocytes. Antioxidants (Basel). 2018; 7(7):95. [DOI:10.3390/antiox7070095]

[28] Anvari D, Jamei R. Evaluation of antioxidant capacity and phenolic content in ethanolic extracts of leaves and flowers of some asteraceae species. Recent Pat Food Nutr Agric. 2018; 9(1):42-9. [PMID]

[29] Ramadan MF, Kroh LW, Mörsel JT. Radical scavenging activity of black cumin (Nigella sativa L.), coriander (Coriandrum sativum L.), and niger (Guizotia abyssinica Cass.) crude seed oils and oil fractions. J Agric Food Chem. 2003; 51(24):6961-9. [DOI:10.1021/jf0346713] [PMID] 
[30] Shahin YR, Elguindy NM, Abdel Bary A, Balbaa M. The protective mechanism of Nigella sativa against diethylnitrosamine-induced hepatocellular carcinoma through its antioxidant effect and EGFR/ ERK1/2 signaling. Environ Toxicol. 2018; 33(8):885-98. [DOI:10.1002/ tox.22574]

[31] Umar S, Zargan J, Umar K, Ahmad S, Katiyar CK, Khan HA. Modulation of the oxidative stress and inflammatory cytokine response by thymoquinone in the collagen induced arthritis in Wistar rats. Chem Biol Interact. 2012; 197(1):40-6. [DOI:10.1016/j.cbi.2012.03.003]

[32] Kang JS, Jeon YJ, Kim HM, Han SH, Yang KH. Inhibition of inducible nitric-oxide synthase expression by silymarin in lipopolysaccharidestimulated macrophages. J Pharmacol Exp Ther. 2002; 302(1):138-44. [DOI:10.1124/jpet.302.1.138] 
This Page Intentionally Left Blank 